\title{
Estudo do envelhecimento e do tratamento térmico de rejuvenescimento da superliga GTD111
}

\author{
Study of the aging and rejuvenation heat \\ treatment of superalloy GTD111
}

André Lucas Merini ${ }^{1}$, Carlos Augusto Silva Oliveira ${ }^{2}$

\footnotetext{
${ }^{1}$ Metalúrgica Merini Ltda - CEP: 89138-000, Ascurra, Santa Catarina, Brasil.

${ }^{2}$ Laboratório de Conformação Mecânica - LABCONF, UFSC, Florianópolis, Santa Catarina, Brasil.

e-mail: andre.merini@merini.com.br, carlos.a@ufsc.br
}

\begin{abstract}
RESUMO
A superliga de níquel GTD111 é comumente utilizada na fabricação de pás de turbinas a gás, destinadas a geração de energia e aplicações aeronáuticas. O presente estudo investiga o comportamento desta superliga "como fornecida" pelo fabricante e previamente tratada termicamente à fim de diminuir o tamanho de $\gamma$ ' e transformá-lo de morfologia esferoidal para cuboide. As amostras foram envelhecidas em laboratório e por fim submetidas ao tratamento térmico de rejuvenescimento com o objetivo de recuperar a microestrutura degradada.

Para cada condição foi avaliada a microdureza, tamanho e percentual de $\gamma^{\prime}$, através de microscopia eletrônica de varredura. Para a etapa de envelhecimento é apresentado um estudo da taxa de coalescimento medida à partir dos resultados de laboratório e a taxa calculada com base na teoria do coalescimento de $\gamma$ '. São apresentadas as duas equações que descrevem a cinética de coalescimento de $\gamma^{\prime}$ deste estudo e realizado um comparativo com outros autores que estudaram a mesma superliga. Os resultados mostraram que as amostras com tratamento térmico de rejuvenescimento prévio têm menor taxa de coalescimento quando comparadas ao estado inicial proveniente do processo de fundição da pá.
\end{abstract}

Palavras-chave: Superliga de níquel. Envelhecimento microestrutural. Tratamento térmico de rejuvenescimento.

\begin{abstract}
The nickel-based superalloy GTD111 is commonly used in the manufacture of gas turbines blades, for applications in power generation and aeronautics. The present study investigates this superalloys's behavior "as provided" from the manufacturer and previously heat treated for $\gamma$ ' size reduction and morphology changing from spheroidal to cuboid. The samples were aged in laboratory and then submitted to the rejuvenation heat treatment with the objective of recovering the degraded microstructure.

For each condition, microhardness, $\gamma^{\prime}$ size and percentage were evaluated using scanning electron microscopy. For the aging stage a study of $\gamma^{\prime}$ coarsening rate measured from experimental results and the calculated rate from $\gamma^{\prime}$ coarsening theory is presented. Two equations that describe the $\gamma^{\prime}$ coarsening kinetics of this study are presented and compared with equations from others authors that studied the same superalloy. The results showed that samples previously heat treated (rejuvenated) have lower coarsening rate when compared to the initial state provided from the blade casting process.
\end{abstract}

Keywords: Nickel superalloy. Microstructural aging. Rejuvenation heat treatment.

\section{INTRODUÇÃO}

Na geração de energia realizada por turbinas a gás, um dos elementos mais importantes são as pás, pois a região onde estão localizadas pode atingir temperatura de operação de aproximadamente $1000{ }^{\circ} \mathrm{C}$, sofrem ação da corrosão e são submetidas à elevadas pressões de trabalho, sendo necessário o emprego de superligas. 
A superliga GTD111 possui aproximadamente $60 \%$ de níquel e teores de cromo e cobalto relativamente elevados. É obtida pelo processo de solidificação direcional e é uma das mais utilizadas na fabricação deste tipo de pá. Devido aos elementos químicos presentes na superliga e seu processo de fabricação, várias fases são observadas na microestrutura [1].

As fases que se formam na microestrutura desta superliga são [2, 3, 4]:

Matriz austenítica $\gamma$ é cúbica de face centrada, à base de níquel e com elevada porcentagem de cobalto, ferro, cromo, molibdênio e tungstênio em solução sólida.

A fase $\gamma^{\prime}\left(\mathrm{Ni}_{3}(\mathrm{Al}, \mathrm{Ti})\right)$ precipitada na matriz $\gamma$ é a principal responsável pela resistência à fluência. Podem formar também eutéticos $\gamma-\gamma^{\prime}$ com variação da morfologia das fases nas regiões interdendríticas.

Pode haver formação de carbonetos primários MC, onde M é um metal e pode ser titânio ou tântalo. Estes carbonetos, normalmente apresentam morfologia irregular, são formados durante a solidificação e distribuídos de maneira heterogênea no material, precipitando tanto nos contornos como no interior do grão.

Os carbonetos secundários $\mathrm{M}_{23} \mathrm{C}_{6}$, normalmente se formam nos contornos de grão, como filmes contínuos. São ricos em cromo e podem ter em solução níquel, molibdênio ou tungstênio.

As fases $\eta$ e $\sigma$ podem se formar, mas em menor porcentagem.

Durante o envelhecimento $\gamma^{\prime}$ coalesce, ocorre precipitação de filmes contínuos de carbonetos secundários $\mathrm{M}_{23} \mathrm{C}_{6}$ nos contornos de grão, os carbonetos primários se dissolvem parcialmente e há formação de fase $\sigma$, o que promove a redução de resistência à fluência.

O coalescimento causa a perda de coerência da fase $\gamma^{\prime} \operatorname{com}$ a matriz $\gamma$, facilitando a movimentação dos contornos de grão. À medida que a coerência diminui, os contornos aumentam a mobilidade e aceleram a cinética de crescimento. Por este motivo, os precipitados $\gamma^{\prime}$ de morfologia cuboide passam a ser esferoidais $[5]$.

O coalescimento de $\gamma^{\prime}$ reduz a energia livre do sistema, pois partículas de menor raio tem maior energia livre molar. Devido ao gradiente de soluto formado, as partículas finas se dissolvem e as grosseiras aumentam seu tamanho [6].

Existem métodos matemáticos para medir o coalescimento de $\gamma^{\prime}$ e prever seu tamanho após determinado tempo de envelhecimento [7].

A equação (1) pode ser usada para determinar a taxa de coalescimento de $\gamma^{\prime}$ [8].

$$
\left(r_{f}^{3}-r_{s}^{3}\right)^{1 / 3}=K . t^{1 / 3}
$$

Onde: t é o tempo em segundos, $\mathrm{r}_{\mathrm{f}}$ é o raio médio final (em nanômetros) de $\gamma^{\prime}$ após o envelhecimento, ou seja, no tempo t. $\mathrm{r}_{\mathrm{s}}$ é o raio médio de $\gamma^{\prime}$ inicial, ou seja, em $\mathrm{t}=0$. K é uma constante.

A equação (2) define o valor da constante K [9].

$$
\ln \left(K^{3} \cdot T\right)=B-\frac{Q}{R \cdot T}
$$

Sendo: Q a energia de ativação para a difusão dos elementos formadores de $\gamma^{\prime}, \mathrm{R}$ a constante dos gases, $\mathrm{T}$ a temperatura absoluta (em Kelvin) e B uma constante [10]. Altos valores de B expressam coalescimento acelerado. O valor de Q para a liga GTD111 é aproximadamente 2,6x105 J/mol [11,12].

Abaixo são apresentadas algumas equações da cinética de coalescimento de $\gamma^{\prime}$ no envelhecimento da superliga GTD111 em diferentes condições iniciais e temperaturas de envelhecimento.

A equação (3) descreve a cinética de coalescimento de $\gamma^{\prime}$ para o experimento realizado por DALEO e WILSON [11]. As amostras tinham tamanho inicial de partículas $\gamma^{\prime}\left(\mathrm{r}_{\mathrm{s}}\right)$ de $0,86 \mu \mathrm{m}$, envelhecimento à $900{ }^{\circ} \mathrm{C}$ por 5000 horas, resultando em tamanho final de partículas $\gamma^{\prime} r_{\mathrm{f}}=1,16 \mu \mathrm{m}$. Com estas informações e com base nas equações (1) e (2), a equação descrita é:

$$
\ln \left(K^{3} \cdot T\right)=38,87-3,267 \times 10^{4} \cdot \frac{1}{T}
$$

A equação (4) foi obtida por TURAZI et al. [2] com referência aos experimentos realizados por CORTEZ [13]. Suas amostras foram envelhecidas à $850{ }^{\circ} \mathrm{C}$ por 2000 horas, tinham $\mathrm{r}_{\mathrm{s}}=0,26 \mu \mathrm{m}$ e após o envelhecimento $r_{f}=0,37 \mu \mathrm{m}$. 


$$
\ln \left(K^{3} . T\right)=37,55-3,267 \times 10^{4} \cdot \frac{1}{T}
$$

A equação (5) foi obtida por TURAZI et al. [2], onde envelheceu amostras à $1000{ }^{\circ} \mathrm{C}$ por 2000 horas, sendo $r_{\mathrm{s}}=0,254 \mu \mathrm{m}$ e $r_{\mathrm{f}}=0,563 \mu \mathrm{m}$.

$$
\ln \left(K^{3} \cdot T\right)=35,84-3,267 \times 10^{4} \cdot \frac{1}{T}
$$

Tratamentos térmicos de rejuvenescimento podem ser realizados para restaurar as propriedades da superliga envelhecida. Estes tratamentos consistem em etapas de solubilização e precipitação, onde o tamanho de $\gamma^{\prime}$ é reduzido e sua distribuição fica mais homogênea. Além disso, a morfologia esferoidal de $\gamma^{\prime}$ passa a ser cuboide, gerando interface $\gamma / \gamma^{\prime}$ coerente. Estes efeitos irão aumentar a resistência a fluência [14, 15].

Para realizar a etapa de solubilização na superliga GTD111, temperaturas superiores a $1150^{\circ} \mathrm{C}$ promovem a dissolução dos precipitados grosseiros que se formaram durante o envelhecimento, evitando que cresçam posteriormente durante a etapa de precipitação $[14,16]$.

\section{MATERIAIS E MÉTODOS}

A Figura 1 mostra uma pá de turbina à gás da superliga GTD111, obtida pelo processo de solidificação direcional, utilizada neste experimento. As amostras foram cortadas em uma máquina de jato de água, evitando transmitir calor à peça. Para avaliar o estado inicial e verificar se haviam diferenças microestruturais e de microdureza, a pá foi dividida em três posições (indicadas na Figura 1 como Pos. 1, Pos. 2 e Pos. 3).

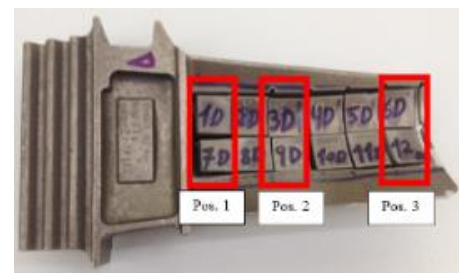

Figura 1: Pá de turbina à gás utilizada no estudo, marcada em três posições: (Pos. 1) base, (Pos. 2) centro, (Pos. 3) ponta.

Parte das amostras foram submetidas à um ciclo de tratamento térmico de rejuvenescimento inicial e a outra parte ficou no estado de fundição, denominando as amostras "com" e "sem" tratamento térmico prévio.

A Tabela 1 mostra a composição química da superliga GTD111 estudada e a Tabela 2 o ciclo de tratamento térmico utilizado nas amostras.

Tabela 1: Composição química da superliga GTD111.

\begin{tabular}{c|c|c|c|c|c|c|c|c|c}
\hline $\mathbf{N i}$ & Cr & Co & Mo & W & Ti & Al & Ta & C & Outros \\
\hline 60 & 14 & 9,5 & 1,5 & 3,8 & 4,8 & 3 & 2,7 & 0,1 & $0,01 \mathrm{~B} / 0,05 \mathrm{Zr}$ \\
\hline
\end{tabular}

Tabela 2: Ciclo de tratamento térmico utilizado no estudo.

\begin{tabular}{c|c|c|c}
\hline 1 SOLUBILIZAÇÃo & 2 $^{\text {a SOLUBILIZAÇÃo }}$ & PRÉ-PRECIPITAÇÃo & PRECIPITAÇÃo \\
\hline $1190^{\circ} \mathrm{C} / 2 \mathrm{~h}$ & $1190^{\circ} \mathrm{C} / 2 \mathrm{~h}$ & $1120^{\circ} \mathrm{C} / 2 \mathrm{~h}$ & $845^{\circ} \mathrm{C} / 24 \mathrm{~h}$ \\
\hline
\end{tabular}

As amostras com e sem tratamento térmico prévio foram envelhecidas à $1000{ }^{\circ} \mathrm{C}$ por 1500 horas, utilizando-se um forno mufla com variação de $\pm 1{ }^{\circ} \mathrm{C}$. Uma amostra de cada condição foi retirada em 250,500 , 750, 1000 e 1500 horas para análises microestruturais e de microdureza. A análise microestrutural avaliou o tamanho e percentual de $\gamma^{\prime}$.

Após a etapa de envelhecimento ser finalizada em 1500 horas, as amostras com e sem tratamento prévio foram submetidas a um tratamento térmico de rejuvenescimento, realizado em forno mufla e com o mesmo ciclo da Tabela 2. Após cada etapa do tratamento térmico de rejuvenescimento, uma amostra de cada condição (com e sem tratamento prévio) foi retirada. Apenas na etapa de precipitação que foram retiradas 
uma amostra de cada condição à cada 6 horas. Foram 14 amostras no total para medir o tamanho e percentual de $\gamma^{\prime}$, bem como microdureza. Vale ressaltar que em todas as etapas do envelhecimento e do tratamento de rejuvenescimento, as amostras foram resfriadas em água à temperatura ambiente.

As análises microestruturais foram realizadas por microscopia eletrônica de varredura (MEV). A preparação das amostras seguiu-se de forma manual com lixas de granulometria até 1200 . No polimento foi utilizada alumina $1 \mu \mathrm{m}$. O ataque químico foi realizado com a solução ASTM E407-170 modificada, que consiste em $61 \%$ de ácido lático $\left(\mathrm{C}_{3} \mathrm{H}_{6} \mathrm{O}_{3}\right), 36 \%$ de ácido nítrico $\left(\mathrm{HNO}_{3}\right), 3 \%$ de ácido fluorídrico $(\mathrm{HF})$.

Para avaliar detalhes da microestrutura e posteriormente medir tamanho e percentual de $\gamma$ ', foi utilizado um MEV modelo Hitachi TM3030, com EDS (espectrometria de dispersão de energia). Para cada condição analisada, foram feitas 5 imagens aleatórias e para as medições de tamanho e percentual de $\gamma$ ' utilizou-se o software "Image J". Algumas amostras apresentaram distribuição bimodal, portanto foi considerada a medição de partículas grosseiras e finas. Para analisar a distribuição bimodal, foram separadas as partículas grosseiras das finas na posição 2 da pá no estado inicial, após 1500 horas de envelhecimento e por fim após processo completo de tratamento térmico de rejuvenescimento, ou seja, após as 24 horas de precipitação.

Para medir a microdureza Vickers foi utilizado o microdurômetro Shimadzu HMV-2, onde foram medidos 8 pontos aleatórios em cada amostra por um tempo de 10 segundos cada endentação e com carga aplicada de $19,614 \mathrm{~N}$.

Para as médias dos resultados apresentados à seguir, utilizou-se um de intervalo de confiança de $95 \%$.

\section{RESULTADOS E DISCUSSÃO}

\subsection{Estado Inicial}

A Figura 2 mostra as microestruturas de cada uma das 3 posições da pá para cada condição: com e sem tratamento térmico de rejuvenescimento prévio. Com base nas imagens foram medidos tamanho e percentual da fase $\gamma^{\prime}$ em cada uma das condições e suas respectivas posições da pá. A condição sem tratamento térmico apresenta uma distribuição bimodal de $\gamma^{\prime}$, ou seja, precipitados mais grosseiros e outros mais finos. Nota-se que o tratamento térmico de rejuvenescimento prévio diminuiu o tamanho das partículas de $\gamma^{\prime}$, bem como alterou sua morfologia, pois antes do tratamento era esferoidal e então passou a ser cuboide após o tratamento, indicando que a interface de $\gamma^{\prime}$ com a matriz $\gamma$ é coerente. Este fato é evidenciado em cada uma das três posições avaliadas na pá.

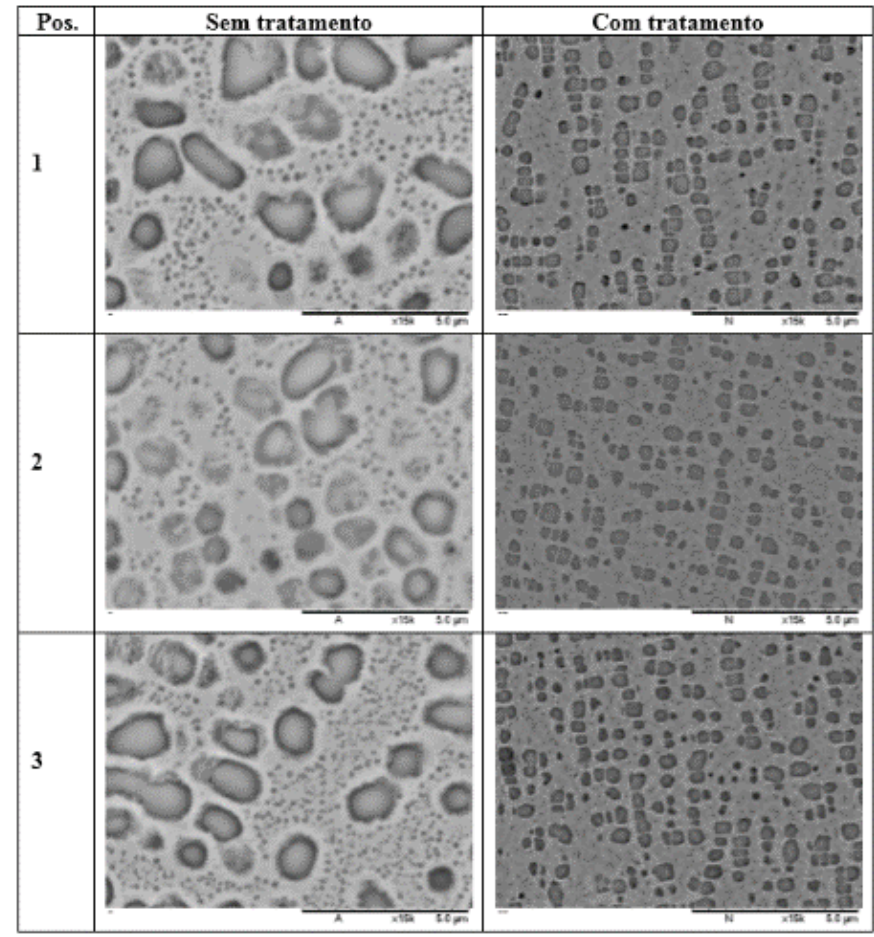

Figura 2: Microestruturas do estado inicial com e sem tratamento térmico em cada uma das três posições da pá. 
As Figuras 3 e 4 mostram respectivamente o tamanho e percentual da fase $\gamma^{\prime}$. Não existe diferença significativa no tamanho de $\gamma^{\prime}$ entre as posições da pá na condição com tratamento térmico prévio. Existem diferenças significativas entre as posições 2 e 3 da pá para a condição sem tratamento térmico prévio e entre as condições sem e com tratamento térmico em todas as posições da pá.

O tamanho médio de $\gamma^{\prime}$ na condição sem tratamento térmico é maior que na condição com tratamento térmico. A dispersão dos resultados é maior na condição sem tratamento, pois o cálculo do tamanho médio é influenciado pela distribuição bimodal. Nota-se que a dispersão é menor na condição com tratamento, pois a porção de partículas finas é muito menor que na condição sem tratamento, conforme mostrado na Figura 3. A Figura 4 mostra que não existe diferença significativa no percentual médio de $\gamma^{\prime}$ entre posições e nem entre condições, o que já era esperado, pois as partículas mudam de tamanho mas seu percentual permanece o mesmo.

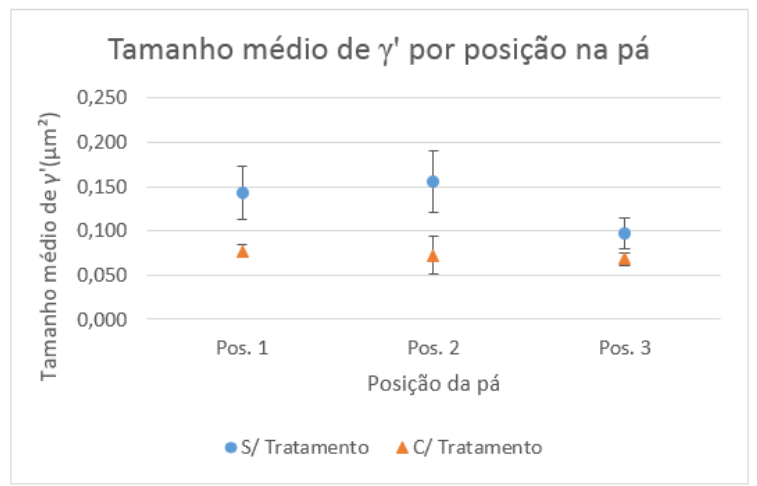

Figura 3: Variação do tamanho médio de $\gamma^{\prime}$ no estado inicial por posição na pá.

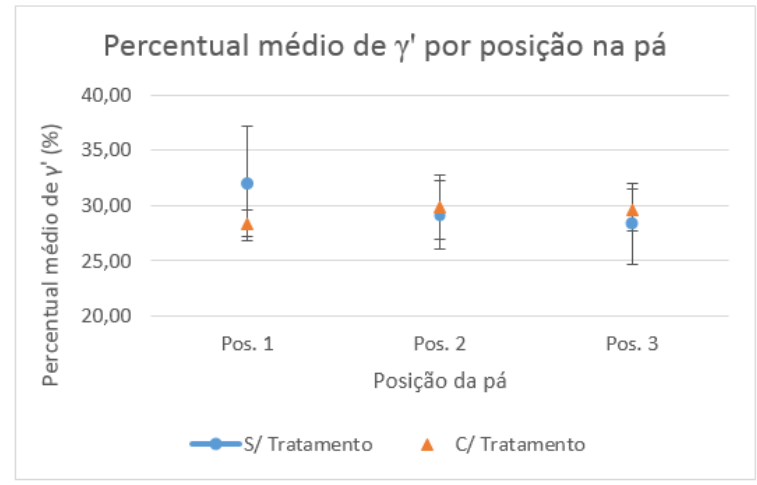

Figura 4: Variação do percentual médio de $\gamma^{\prime}$ no estado inicial por posição na pá.

Para analisar a distribuição bimodal a medição do tamanho médio de $\gamma$ ' assumiu-se que $\gamma$ ' "grosseira" tinha tamanho acima de $0,04 \mu \mathrm{m}^{2}$. O mesmo valor foi adotado por CORTEZ [13].

A condição sem tratamento térmico apresentou tamanho médio de $\gamma^{\prime}$ grosseira igual a $0,364 \mu \mathrm{m}^{2}$, com intervalo de confiança igual a $\pm 0,086 \mu \mathrm{m}^{2}$. O percentual médio de partículas grosserias de $\gamma$ ' foi de $29,78 \%$ (I.C. $= \pm 3,54 \%$ ). Já a condição com tratamento térmico apresentou tamanho médio igual a $0,205 \mu \mathrm{m}^{2}$ (I.C. $= \pm 0,049 \mu \mathrm{m}^{2}$ ) e percentual médio igual a 33,68 \% (I.C. $= \pm 3,12 \%$ ).

O tamanho médio de partículas grosserias de $\gamma^{\prime}$ em ambas as condições de tratamento é maior em relação ao mostrado na Figura 4, pois neste caso as partículas finas não estão influenciando para diminuir a média. Mesmo assim, permanece a diferença significativa entre as condições de tratamento, sendo o tamanho médio da condição sem tratamento significativamente maior que a condição com tratamento.

Não há diferença significativa no percentual de partículas grosseiras de $\gamma^{\prime}$ entre as condições com e sem tratamento térmico. Se os resultados forem comparados com o gráfico da Figura 4, também não existe diferença significativa.

Foram observados carbonetos primários nas condições com e sem tratamento prévio. A Figura 5 mostra um desses carbonetos para a condição sem tratamento prévio e a Tabela 3 mostra a composição química 
obtida por EDS deste carboneto. Os metais Titânio e Tântalo apresentam percentual relativamente elevados, indicando serem carbonetos primários MC assim como citado por TURAZI et al. [2] e DONACHIE e DONACHIE [3].

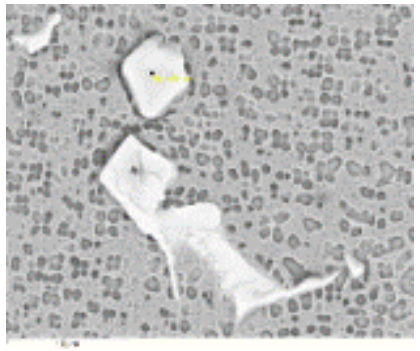

Figura 5: Região de análise por EDS com a presença de carbonetos primários formados na solidificação.

Tabela 3: Composição química do carboneto primário MC.

\begin{tabular}{l|c}
\hline ELEMENTO & PESO (\%) \\
\hline Carbono & 31,249 \\
\hline Oxigênio & 6,786 \\
\hline Enxofre & 0,493 \\
\hline Titânio & 20,438 \\
\hline Cromo & 1,900 \\
\hline Níquel & 5,298 \\
\hline Tântalo & 27,326 \\
\hline Tungstênio & 6,510 \\
\hline
\end{tabular}

A Figura 6 mostra as médias de microdureza de cada posição da pá nas condições com e sem tratamento térmico prévio. As três posições sem tratamento prévio apresentam microdureza média de 451 HV2, sendo que a diferença entre as posições não é significativa. Nota-se que para as mesmas posições, quando as amostras são submetidas ao tratamento térmico de rejuvenescimento, a microdureza aumenta em média $23 \%$, ou seja, passa para 554 HV2. Este aumento de microdureza é devido a coerência entre $\gamma / \gamma^{\prime}$, redução do tamanho e menor caminho médio entre os precipitados $\gamma^{\prime}$ na condição com tratamento, sendo significativamente maior que a microdureza média da condição sem tratamento. A morfologia cuboide aumenta a barreira para as discordâncias e com isso dificulta sua mobilidade, explicando o maior resultado de microdureza [2].

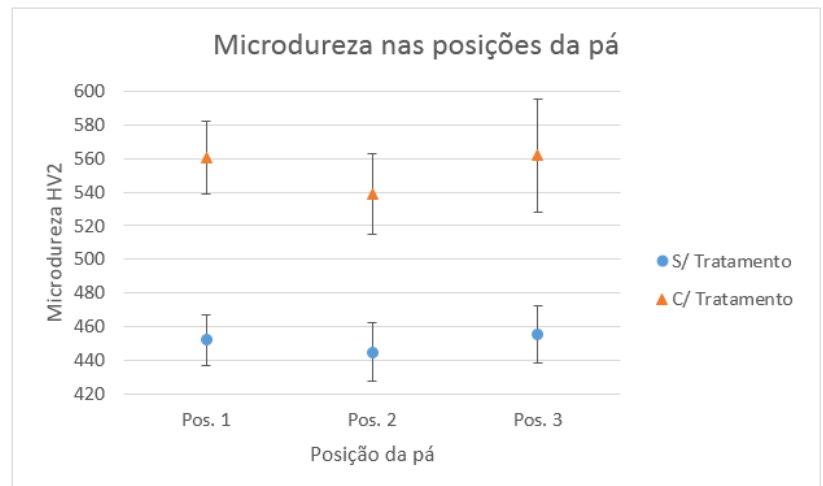

Figura 6: Variação da microdureza do estado inicial para as 3 posições da pá nas condições com e sem tratamento térmico.

\subsection{Envelhecimento}

Após a avaliação do estado inicial, as amostras foram submetidas ao envelhecimento. As Figuras 7 a 9 mostram respectivamente as microestruturas de cada etapa do envelhecimento e condição de tratamento térmico 
prévio, a variação de tamanho e percentual de $\gamma^{\prime}$. Em $250 \mathrm{~h}$ verifica-se que nas amostras com tratamento térmico $\gamma^{\prime}$ permanece com morfologia cuboide, o que é indicado para resistência e retardamento à degradação conforme citado por CORTEZ [13] e KOUNTRAS [7]. Nota-se que para ambas as condições, ao longo do envelhecimento, $\gamma^{\prime}$ aumenta de tamanho e na condição tratada, a morfologia cuboide muda para esferoidal, devido à perda de coerência da interface $\gamma^{\prime}$ com a matriz $\gamma$ [17]. Portanto, o crescimento do precipitado $\gamma^{\prime}$ é acelerado devido a perda da capacidade de ancorar discordâncias.

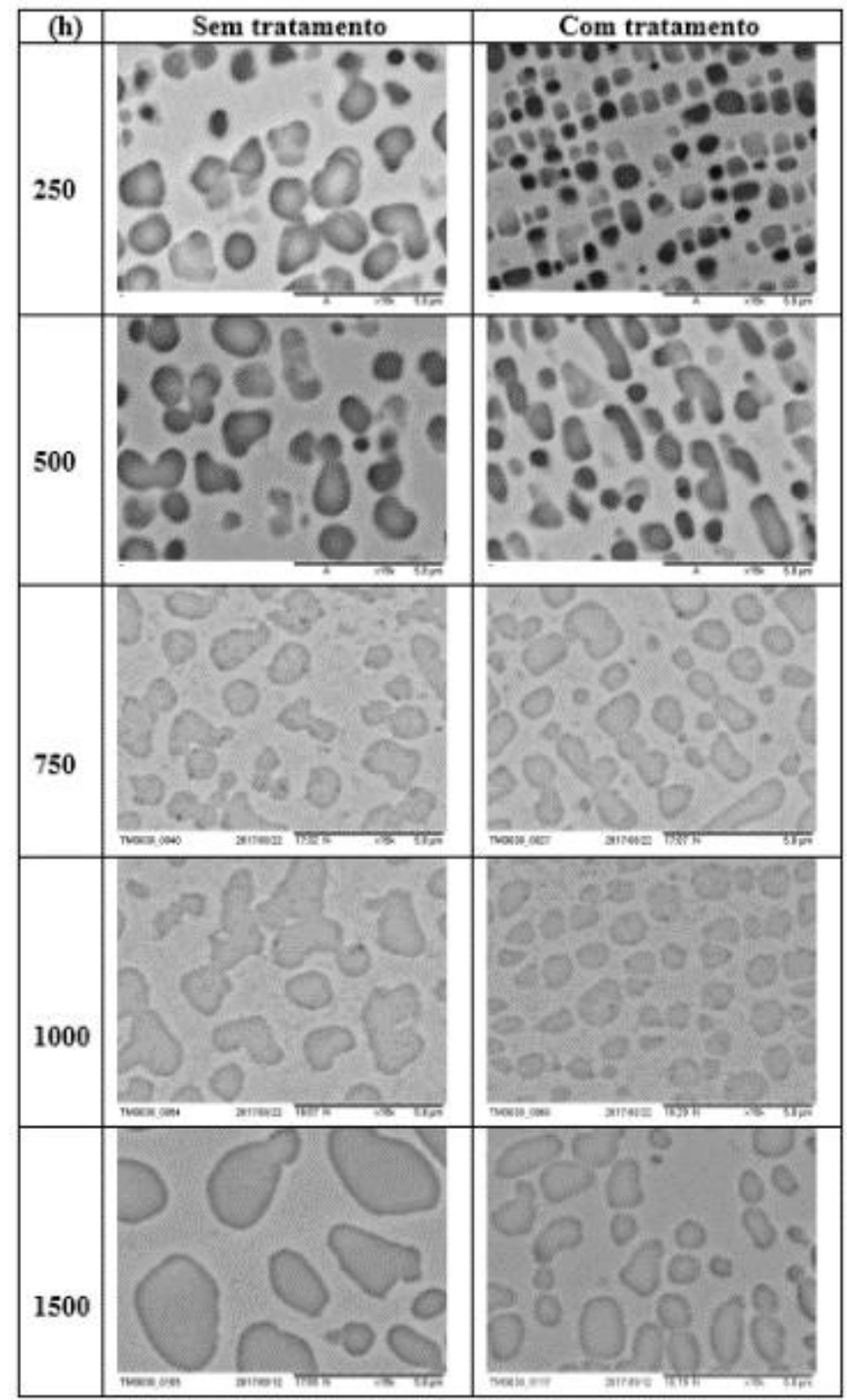

Figura 7: Microestrutura das amostras com e sem tratamento térmico prévio envelhecidas em laboratório à $1000{ }^{\circ} \mathrm{C}$.

O tamanho médio de $\gamma^{\prime}$ é significativamente maior para a condição sem tratamento até $1000 \mathrm{~h}$ de envelhecimento, porém quando atinge 1500 h, a diferença não é mais significativa. A perda de significância da diferença de tamanho de $\gamma^{\prime}$ se deve ao fato de que a dispersão do tamanho dos precipitados das amostras sem tratamento à partir de $1000 \mathrm{~h}$ são relativamente maiores, o que reforça que em função da distribuição bimodal os precipitados mais finos dissolveram para os precipitados grosseiros coalescerem, portanto a amplitude de variação da condição sem tratamento térmico prévio encontra a amplitude de variação da condição com tratamento térmico. Mesmo assim, a linha de tendência é clara e mostra que o tamanho médio da condição sem tratamento térmico é maior que a tratada.

A linha de tendência mostra que a condição com tratamento resulta em um percentual de $\gamma^{\prime}$ maior que a condição sem tratamento, porém é possível notar elevada dispersão nos resultados e em $750 \mathrm{~h}$ a condição se 
inverte. Embora existam diferenças nas análises do percentual de $\gamma^{\prime}$, não é provável de ter ocorrido, pois na condição inicial era $30 \%$ e o envelhecimento não reduz o percentual. Possivelmente partículas muito finas não tenham sido observadas devido à resolução do microscópio. Pode-se dizer que não existe diferença significativa entre as amostras com e sem tratamento e nem em função do tempo de envelhecimento.

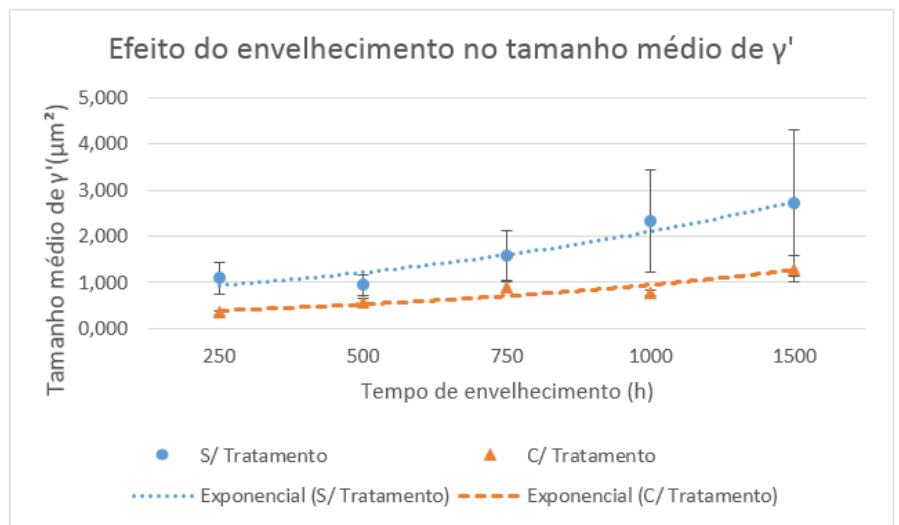

Figura 8: Variação do tamanho médio de $\gamma^{\prime}$ com o tempo de envelhecimento para as amostras com e sem tratamento térmico prévio.

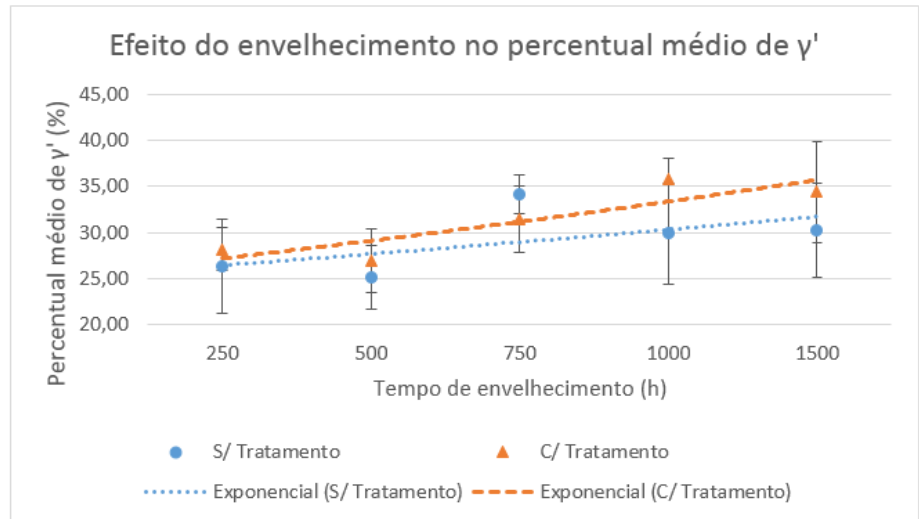

Figura 9: Variação do percentual de $\gamma^{\prime}$ com o tempo de envelhecimento para as amostras com e sem tratamento térmico prévio.

Após 1500 horas de envelhecimento as partículas grosseiras de $\gamma^{\prime}$ nas amostras sem tratamento térmico apresentaram tamanho médio igual a $2,824 \mu \mathrm{m}^{2}\left(\mathrm{I} . \mathrm{C} . \quad= \pm 1,581 \mu \mathrm{m}^{2}\right)$ e percentual médio de partículas grosseiras de $\gamma^{\prime}$ igual a $30,99 \%$ (I.C. $= \pm 4,59 \%$ ). As amostras com tratamento prévio apresentaram tamanho igual a $1,404 \mu \mathrm{m}^{2}\left(\right.$ I.C. $\left.= \pm 0,417 \mu \mathrm{m}^{2}\right)$ e percentual igual a $35,46 \%$ (I.C. $= \pm 5,57 \%$ ). Nota-se que a dispersão do resultado da amostra sem tratamento térmico prévio é maior que na condição com tratamento prévio, porém a diferença não é significativa. Observa-se que o tamanho médio das partículas grosseiras de $\gamma^{\prime}$ é muito próximo do valor obtido na Figura 8, que não considera as partículas finas. Isto é devido ao pequeno efeito no tamanho total das partículas de $\gamma^{\prime}$ menores que $0,04 \mu \mathrm{m}^{2}$.

A diferença do percentual médio de partículas grosseiras de $\gamma^{\prime}$ entre a condição com e sem tratamento térmico prévio não é significativa mesmo que sejam analisadas apenas partículas grosseiras, porém se o resultado for comparado com a as Figura 9, existe um aumento na ordem de $2 \%$ no percentual médio. Estes resultados são próximos aos obtidos por CORTEZ [13].

A Figura 10 mostra o efeito do tempo de envelhecimento na microdureza das amostras na temperatura de $1000{ }^{\circ} \mathrm{C}$, onde a microdureza decresce em função do tempo. Em $250 \mathrm{~h}$ a diferença não é significativa, porém no intervalo de $500 \mathrm{~h}$ a $1000 \mathrm{~h}$ a diferença é significativa. As linhas de tendência mostram que a redução de microdureza das amostras com tratamento térmico é menor do que a condição sem tratamento.

No estado inicial a diferença média entre a microdureza das amostras era $23 \%$ e eram significativamente diferentes entre si. Após $1500 \mathrm{~h}$ de envelhecimento as amostras com e sem tratamento tem sua microdureza reduzida e não existem diferenças significativas entre si. Na microestrutura da amostra com tratamento, a morfologia de $\gamma^{\prime}$ muda de cuboide para esferoidal, causada pela perda de coerência de $\gamma^{\prime}$ com a matriz $\gamma$. 
Esta perda de coerência tem menor efeito em ancorar as discordâncias e portanto explica a redução da microdureza.

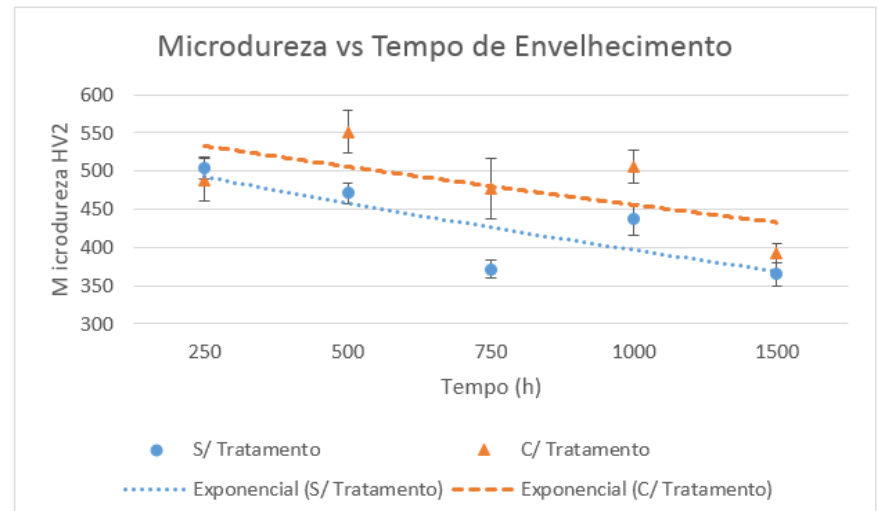

Figura 10: Variação da microdureza com o tempo de envelhecimento das amostras com e sem tratamento térmico prévio.

\subsection{Determinação da cinética de coalescimento de $\gamma^{\prime}$ da superliga GTD111}

Utilizando os resultados do tamanho de $\gamma^{\prime}$ durante a etapa de envelhecimento é possível determinar a equação que descreve a cinética de coalescimento de $\gamma^{\prime}$ durante o envelhecimento. No cálculo da cinética do coalescimento, $\gamma$ ' foi considerada circular, mesmo que para as amostras com tratamento, $\gamma^{\prime}$ seja cuboide. Calculou-se a média das áreas das três posições inicialmente medidas: $r_{\mathrm{s}}=205 \mathrm{~nm}$ nas amostras sem tratamento prévio e $\mathrm{r}_{\mathrm{s}}=$ $152 \mathrm{~nm}$ nas amostras com tratamento prévio. O raio final dos precipitados $\gamma^{\prime}$ após $1500 \mathrm{~h}$ de envelhecimento foram obtidos à partir das medições de área nas micrografias de cada etapa do envelhecimento.

Ao utilizar a equação (1) é possível encontrar o valor da constante K, sendo para as amostras sem e com tratamento prévio $\mathrm{K}^{3}=147,29 \mathrm{~nm}^{3} / \mathrm{s} \mathrm{e}^{3}=42,06 \mathrm{~nm}^{3} / \mathrm{s}$, respectivamente. Vale ressaltar que para se determinar a cinética de coalescimento considerou-se os tamanhos médios de partículas finas e grosseiras. A partir do valor de K médio, obtém-se os valores de variação do tamanho de $\gamma^{\prime}$ em função do tempo utilizando a equação (1). Não foram obtidas amostras para o tempo de $1250 \mathrm{~h}$, entretanto é possível fazer a previsão de tamanho de $\gamma$ '.

A Figura 11 mostra a variação do tamanho medido e calculado, onde é possível notar que os valores são próximos, isto é, a linha que corresponde ao valor calculado está próxima dos pontos que foram medidos, indicando que os métodos matemáticos podem ser aplicados. A inclinação das duas retas mostra que o tamanho de $\gamma^{\prime}$ aumenta em função do tempo na temperatura de $1000{ }^{\circ} \mathrm{C}$ e que as amostras sem tratamento possuem taxa de coalescimento maior que as amostras sem tratamento. As amostras com tratamento térmico prévio possuem taxa de coalescimento menor pois a morfologia inicial cuboide de $\gamma^{\prime}$, que indica coerência da interface $\gamma / \gamma^{\prime}$, retarda o coalescimento de $\gamma^{\prime}[2,7]$.

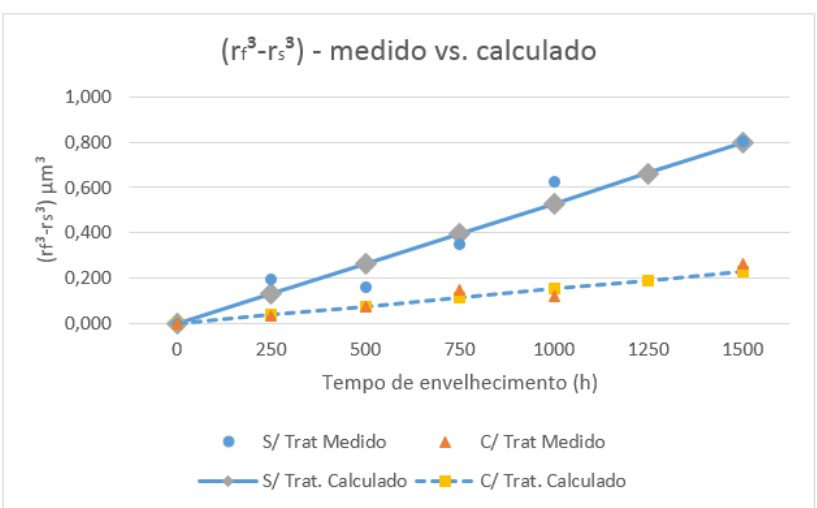

Figura 11: Variação do tamanho de $\gamma^{\prime}$ medido e calculado em função do tempo de envelhecimento.

Para determinar a equação que descreve a cinética de envelhecimento do experimento em questão, considerou-se a temperatura de $1000{ }^{\circ} \mathrm{C}(1273 \mathrm{~K})$, os valores de $\mathrm{K}$ acima mencionados e à partir da equação (2) pode-se encontrar o valor da constante B para as amostras sem e com tratamento prévio, sendo 37,81 e 


\section{6,55 respectivamente.}

As equações (6) e (7) determinam a cinética de coalescimento de $\gamma^{\prime}$ para as condições sem e com tratamento, respectivamente. Os resultados das equações (3) a (7) são comparados na Figura 12, em um intervalo de temperatura entre 1000 e $850^{\circ} \mathrm{C}$. É possível notar a proximidade de resultados entre a condição sem tratamento deste experimento com as de CORTEZ [13] citado por TURAZI et al. [2]. Esta proximidade é devida aos valores da constante B serem muito próximos entre as equações (4) e (6). Os valores da constante B entre as equações (3) a (7) diferem pois o tamanho inicial e a morfologia dos precipitados $\gamma^{\prime}$ não são iguais, resultando em taxas de coalescimento diferentes.

Quanto maior o valor da constante B, mais acelerado é o coalescimento [2, 7, 11, 12]. Portanto, ao comparar as condições com e sem tratamento prévio na mesma temperatura de envelhecimento, a condição com tratamento prévio possui taxa de coalescimento menor que a sem tratamento prévio, devido a morfologia cuboide atrasar o efeito do coalescimento que promove a esferoidização do precipitado $\gamma^{\prime}$. Na morfologia cuboide a interface entre precipitado e matriz é plana, o que restringe a mobilidade do precipitado e por isso a cinética de crescimento é menor. Com base nisto o tempo em serviço da superliga GTD111 com tratamento térmico de rejuvenescimento prévio poderia ser maior que a condição obtida após fundição, ou seja, sem tratamento.

$$
\begin{aligned}
& \ln \left(K^{3} \cdot T\right)=37,81-3,267 \times 10^{4} \cdot \frac{1}{T} \\
& \ln \left(K^{3} \cdot T\right)=36,55-3,267 \times 10^{4} \cdot \frac{1}{T}
\end{aligned}
$$

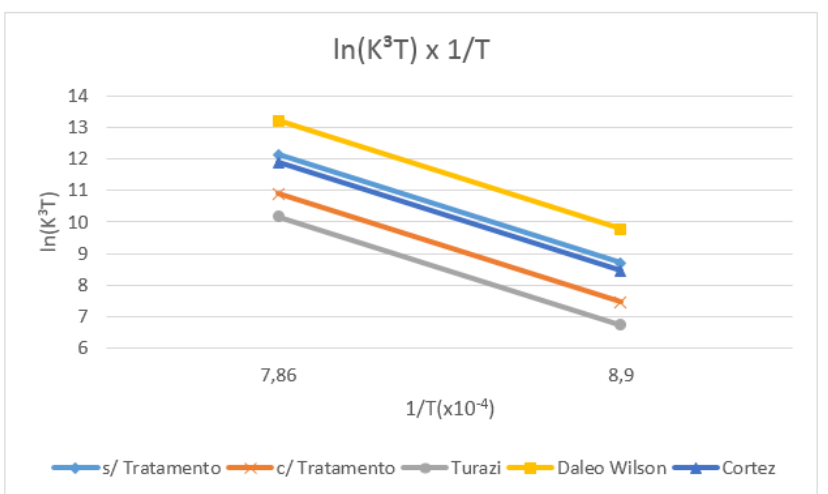

Figura 12: Variação de $\ln \left(\mathrm{K}^{3} \mathrm{~T}\right)$ em função de 1/T para o coalescimento de $\gamma^{\prime}$ durante o envelhecimento, comparando as equações obtidas no presente estudo com as da literatura.

\subsection{Tratamento térmico de rejuvenescimento}

Após o envelhecimento de $1500 \mathrm{~h}$, as amostras com e sem tratamento térmico prévio foram submetidas ao tratamento térmico de rejuvenescimento, utilizando o mesmo ciclo mostrado na Tabela 2. Por questões de nomenclatura, as amostras continuam sendo denominadas com e sem tratamento nas figuras, referindo ao estado inicial, ou seja, com ou sem tratamento prévio. Todas as amostras foram tratadas ao mesmo tempo, com o objetivo de eliminar ou reduzir possíveis erros.

A Figura 13 mostra as microestruturas obtidas das amostras com e sem tratamento térmico prévio em cada etapa do tratamento de rejuvenescimento. Nas duas etapas de solubilização, à $1190{ }^{\circ} \mathrm{C}$, nota-se que o tamanho de $\gamma^{\prime}$ é menor do que na condição envelhecida, na Figura 7, isto porque os mesmos foram dissolvidos. Na segunda solubilização, também à $1190^{\circ} \mathrm{C}$ e por $2 \mathrm{~h}$, $\gamma^{\prime}$ é ainda mais fino.

Em ambas as condições de tratamento, algumas regiões apresentaram $\gamma^{\prime}$ coalescido com tamanho próximo ao medido após $1500 \mathrm{~h}$ de envelhecimento, evidenciando que alguns precipitados $\gamma^{\prime}$ não solubilizaram por completo. A Figura 14 mostra a microestrutura da amostra sem tratamento prévio após segunda solubilização, sendo que esta condição foi a que apresentou mais regiões com $\gamma^{\prime}$ grosseira. Conforme citado por WANGYAO et al. [14] as partículas de $\gamma^{\prime}$ não solubilizadas irão coalescer durante a etapa de precipitação no rejuvenescimento. Portanto, na microestrutura após tratamento térmico de rejuvenescimento, estarão presentes partículas de $\gamma^{\prime}$ grosseiras e com morfologia esferoidal, havendo a tendência de coalescer mais rapida- 
mente em serviço e portanto reduzir a resistência à fluência [16].

As Figuras 15 e 16 mostram respectivamente a variação do tamanho e do percentual de $\gamma^{\prime}$ em cada etapa do tratamento térmico de rejuvenescimento. Não foi observada diferença significativa no tamanho médio de $\gamma^{\prime}$ entre as amostras com e sem tratamento prévio. É possível verificar que à partir de $12 \mathrm{~h}$ de precipitação, mesmo que a distribuição seja bimodal, ambas as condições apresentam menor dispersão dos resultados, ou seja, o tamanho e a distribuição mais homogênea de $\gamma^{\prime}$ na microestrutura acontece de maneira significativa entre 12 e $24 \mathrm{~h}$ de precipitação.

A diferença do percentual de partículas de $\gamma^{\prime}$ entre as condições com e sem tratamento prévio não são significativas. Na solubilização o percentual de partículas é menor pois as partículas são dissolvidas. Após a precipitação o percentual médio de $\gamma^{\prime}$ resulta entre 25 e $30 \%$, valor similar ao encontrado no estado inicial e ao final do envelhecimento.

O tamanho médio de partículas grosseiras de $\gamma^{\prime}$ para as condições sem e com tratamento térmico prévio é igual a $0,139 \mu \mathrm{m}^{2}\left(\right.$ I.C. $\left.= \pm 0.014 \mu \mathrm{m}^{2}\right)$ e $0,164 \mu \mathrm{m}^{2}\left(\right.$ I.C. $\left.= \pm 0.026 \mu \mathrm{m}^{2}\right)$, respectivamente. O tamanho médio é maior quando são analisadas apenas partículas grosseiras. Isto é esperado, pois as partículas menores que $0,04 \mu \mathrm{m}^{2}$ estão desconsideradas e portanto resultam num valor de média maior. O tamanho médio de $\gamma^{\prime}$ é significativamente menor que na condição envelhecida após $1500 \mathrm{~h}$ e ao comparar os resultados do estado inicial e após o rejuvenescimento, a diferença no tamanho médio de $\gamma^{\prime}$ não é significante. Isto indica que o rejuvenescimento fez com que $\gamma^{\prime}$ tenha retornado ao tamanho próximo do medido no estado inicial.

O percentual de partículas grosseiras de $\gamma^{\prime}$ das amostras sem e com tratamento térmico prévio é igual a $26,50 \%$ (I.C. $= \pm 4,31 \%$ ) e $33,27 \%$ (I.C. $= \pm 2,87 \%$ ), respectivamente. Também não existe diferença significativa no percentual de partículas grosseiras de $\gamma^{\prime}$ quando comparados: o estado inicial, após envelhecimento e após tratamento térmico de rejuvenescimento entre as condições com e sem tratamento térmico prévio. Sendo assim, nenhuma das etapas do experimento modificou o percentual de $\gamma$ '.

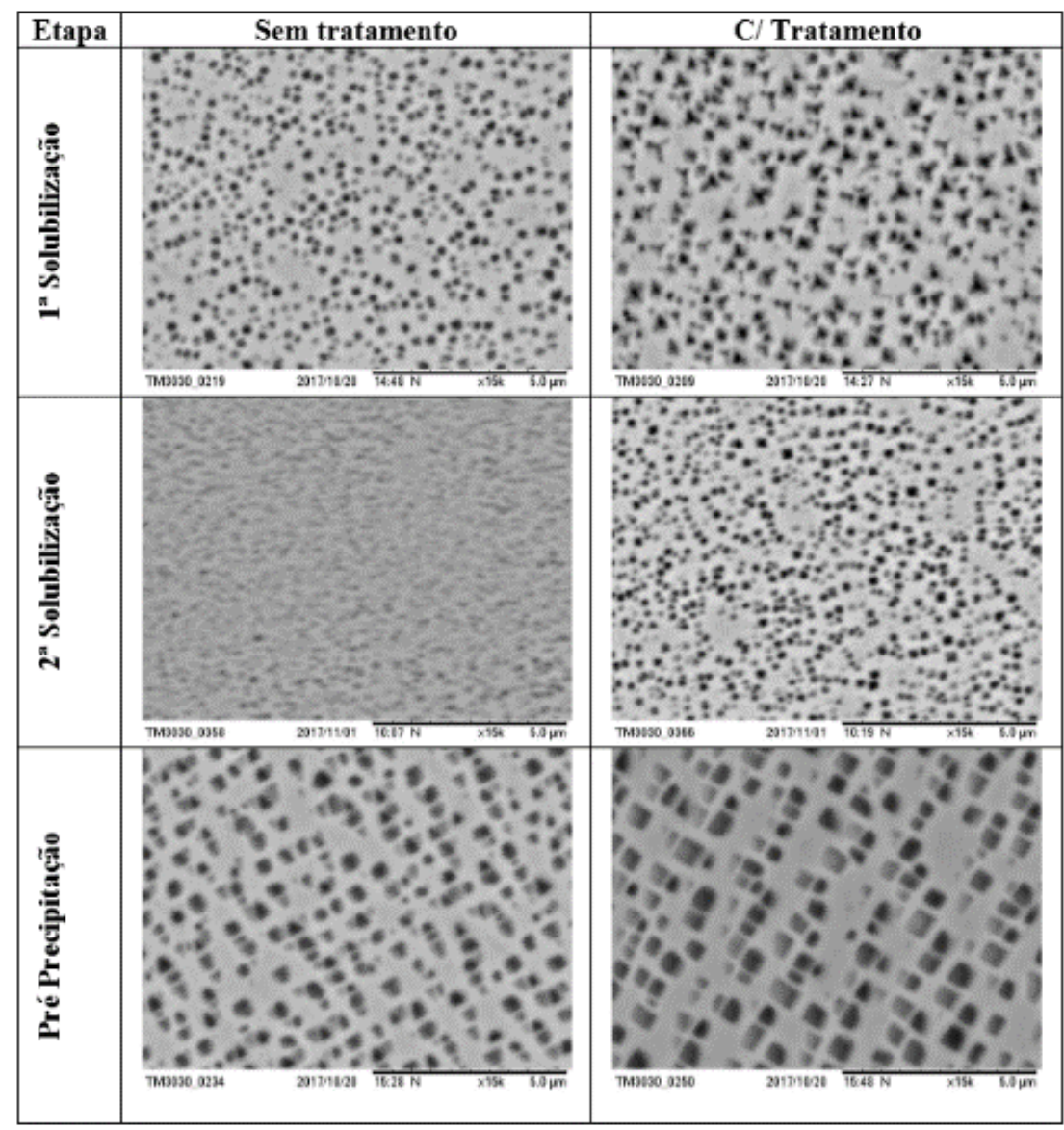




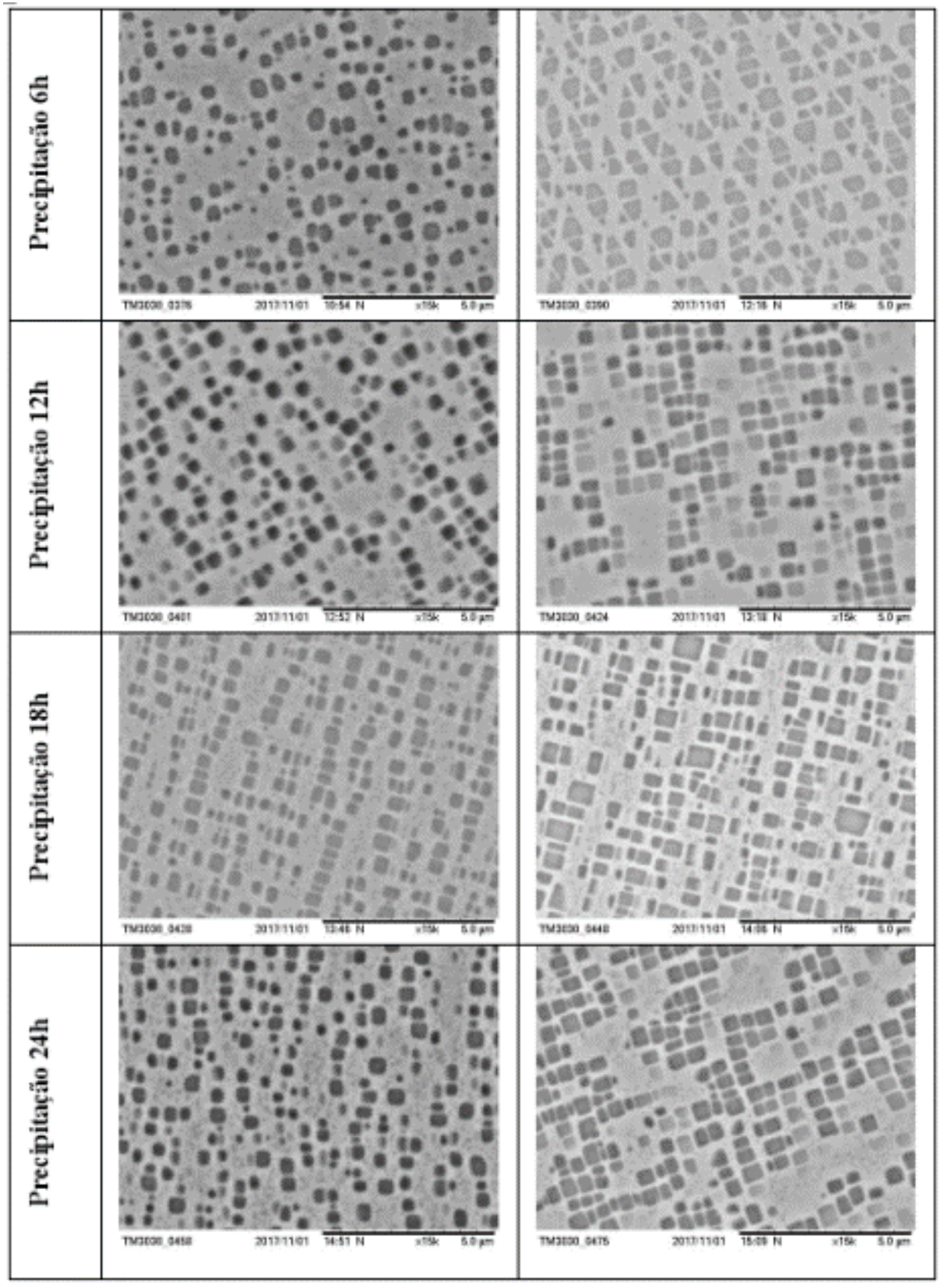

Figura 13: Microestruturas obtidas em cada etapa do tratamento térmico de rejuvenescimento para as amostras sem e com tratamento térmico de rejuvenescimento prévio.

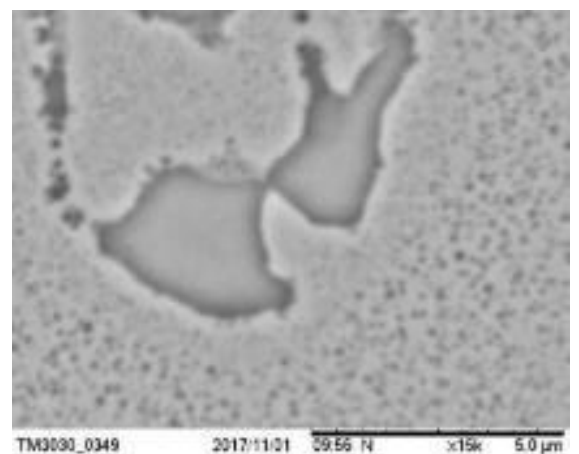

Figura 14: Microestrutura da amostra sem tratamento após segunda solubilização apresentando região com partículas de $\gamma$ ' não solubilizadas totalmente. 


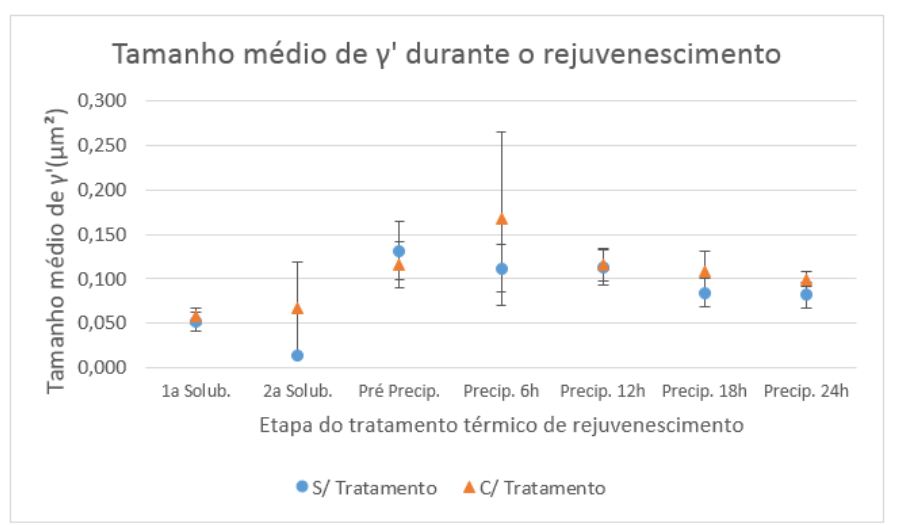

Figura 15: Variação do tamanho médio de $\gamma^{\prime}$ durante o tratamento térmico de rejuvenescimento.

Percentual médio de $\gamma^{\prime}$ durante o rejuvenescimento

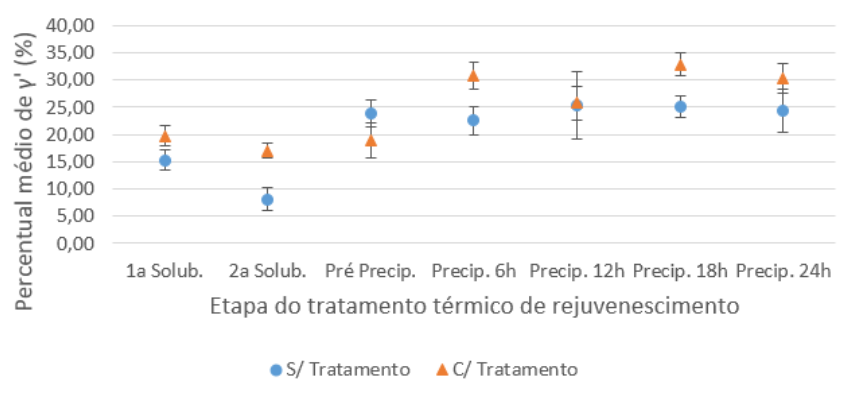

Figura 16: Variação do percentual médio de $\gamma^{\prime}$ durante o tratamento térmico de rejuvenescimento.

A Figura 17 mostra os resultados de microdureza média das amostras com e sem tratamento térmico prévio em cada etapa do tratamento de rejuvenescimento. Com exceção da segunda etapa de solubilização, nas demais etapas a microdureza das amostras com tratamento prévio foi significativamente menor que as amostras sem tratamento prévio.

A condição final das amostras é determinada após 24 h de precipitação, ou seja, após a execução completa do ciclo. A microdureza média das amostras sem tratamento prévio é $6 \%$ maior que a amostra com tratamento prévio, portanto menor que a diferença apresentada no estado inicial que era $23 \%$.

Comparando a microdureza após tratamento térmico de rejuvenescimento com a condição após 1500 $\mathrm{h}$ de envelhecimento observa-se que a amostra sem tratamento prévio aumenta sua microdureza média em $36 \%$ e a amostra com tratamento prévio $19 \%$. TURAZI [16] também apresentou resultados de microdureza similares ao realizar o mesmo ciclo de tratamento térmico de rejuvenescimento do presente estudo.

Ao comparar a amostra sem tratamento prévio após ter sido envelhecida e posteriormente rejuvenescida com seu estado inicial (fundida), a microdureza média foi $10 \%$ superior. Este aumento de microdureza é devido a $\gamma^{\prime}$ no estado inicial ser maior e possuir morfologia esferoidal.

O aumento de microdureza após o rejuvenescimento é devido a restauração da coerência da interface $\gamma / \gamma^{\prime}$, evidenciada pela morfologia cuboide de $\gamma^{\prime}$, pela redução no tamanho e maior homogeneidade na distribuição de $\gamma^{\prime}$ na matriz quando comparada com as amostras envelhecidas por $1500 \mathrm{~h}[13,14,16]$. Conforme citado anteriormente, na morfologia cuboide a interface entre matriz e precipitado é plana, dificultando a mobilidade do precipitado e aumentando a capacidade de ancorar discordâncias. Esta característica proporciona maior microdureza. 


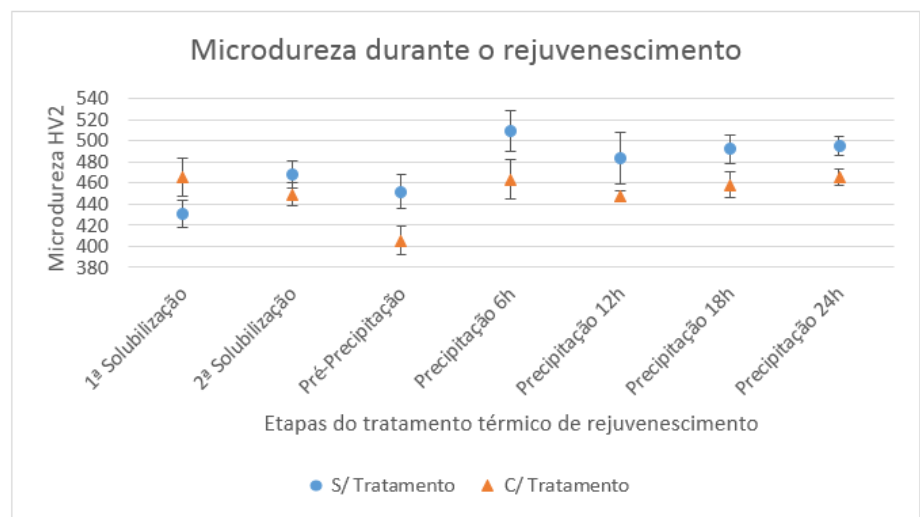

Figura 17: Variação da microdureza em cada etapa de tratamento térmico de rejuvenescimento para amostras com e sem tratamento térmico prévio.

\section{CONCLUSÕES}

A pá da turbina à gás no estado inicial, após fundição e sem tratamento térmico de rejuvenescimento, apresentou diferença de tamanho médio de partículas $\gamma$ ' ao longo de sua extensão. Os menores valores médios estão localizados na ponta da pá.

O tratamento térmico de rejuvenescimento prévio reduz a diferença do tamanho médio de $\gamma^{\prime}$ entre as posições da pá e torna a diferença não significativa. O tamanho médio de partículas $\gamma^{\prime}$ é $55 \%$ menor que no estado inicial após fundição.

A microestrutura das amostras no estado inicial sem tratamento térmico de rejuvenescimento prévio apresenta $\gamma^{\prime}$ com morfologia esferoidal, ao contrário das amostras com tratamento térmico prévio que apresenta $\gamma^{\prime}$ com morfologia cuboide.

No estado inicial, a microdureza média das três posições da pá é $23 \%$ maior nas amostras com tratamento prévio do que nas amostras sem tratamento prévio, devido à morfologia, ao menor tamanho e distribuição mais homogênea de $\gamma^{\prime}$.

A equação da cinética de coalescimento de $\gamma^{\prime}$ da superliga GTD111 pode ser expressa pela equação (8), sendo o valor da constante B igual a 37,81 e 36,55 nas amostras sem e com tratamento prévio, respectivamente. Esta equação auxilia a prever o tamanho do precipitado $\gamma$ ' sabendo a temperatura em que o material vai operar e o tamanho de $\gamma^{\prime}$ inicial. Também auxilia a determinar o momento do qual a turbina deve parar de operar para realizar tratamento térmico de rejuvenescimento ou substituição das pás.

$$
\ln \left(K^{3} . T\right)=B-3,267 \times 10^{4} \cdot \frac{1}{T}
$$

As amostras que foram submetidas ao tratamento térmico de rejuvenescimento antes de serem envelhecidas apresentam menor taxa de coalescimento do que as amostras sem tratamento, evidenciado pelo menor valor da constante B. Portanto, as amostras com tratamento prévio suportam maior tempo de exposição ao envelhecimento.

$\mathrm{O}$ tratamento térmico de rejuvenescimento recupera a microestrutura degradada pelo envelhecimento, evidenciado pela recuperação da perda de coerência dos precipitados $\gamma^{\prime}$ com a matriz $\gamma$. O tamanho de $\gamma^{\prime}$ é diminuído e retorna à morfologia cuboide, apresentando valores similares à condição inicial com tratamento térmico prévio, inclusive para os resultados de microdureza.

Mesmo após o envelhecimento, as amostras sem tratamento térmico prévio, após o tratamento térmico de rejuvenescimento final apresentaram tamanho de $\gamma^{\prime}$ menor e microdureza $10 \%$ maior em relação ao estado inicial (após fundição).

\section{AGRADECIMENTOS}

Os autores agradecem a equipe de estagiários do LABCONF (Laboratório de conformação mecânica) e LMP (Laboratório de mecânica de precisão) da Universidade Federal de Santa Catarina e ao CNPq pela bolsa de pesquisador de C.A.S Oliveira. 


\section{BIBLIOGRAFIA}

[1] Complex Nickel Alloys. IRELAND ALLOYS. http://www.ireland-alloys.co.uk/tabid/108/Default.aspx. Acessado em 20/11/2017.

[2] TURAZI, A., OLIVEIRA, C.A.S., BOHÓRQUEZ, C.E.N., et al., "Metodologia para avaliação da degradação Microestrutural de Pás de Turbinas a Gás", In: IX CITENEL, 2017.

[3] DONACHIE, M.J., DONACHIE, S.J. Superalloys: A technical guide. 2 ed., Materials Park. OH. ASM International, 2002.

[4] EL-BAGOURY, N. "Microstructure and mechanical properties of aged nickel base superalloy," Scholars Research Library, Archives of Applied Science Research, v. 3, n.2, pp. 266-276, 2011.

[5] REED, R. C. The superalloys: fundamentals and applications. Cambridge University Press, 2006.

[6] DURAND-CHARRE, M. The microstructure of superalloys. Netherlands, Gordon \& Breach Science Publishers. Amsterdam, 1997.

[7] KOUNTRAS, A. Metallographic study of gamma - gamma prime structure in the Ni-based superalloy GTD-111. Degree Ph.D, MIT, 2004.

[8] CHELLMAN, D.J., ARDELL, A.J. "The coarsening of gamma prime precipitates at large volume fractions”, Acta Metallurgica, pp. 577-588, 1974.

[9] STEVENS, R.A., FLEWITT, P.E.J. "The effects of $\gamma$ ' precipitate coarsening during isothermal aging and creep of the nickel-base superalloy IN-738”, Materials Science and Engineering., v.37, pp. 237-247, 1979.

[10] LVOVA, E., NORSWORTHY, D. "Influence of previous operational cycle on the microstructure of rejuvenated Ni-base superalloy gas turbine blades after their return to service", In: Proc. $200029^{\text {th }}$ Turbomachinery Symposium, pp. 113-124.

[11] DALEO, J.A., WILSON, J.R. "GTD-111 Alloy Material Study", Journal of Engineering for Gas Turbines and Power, pp. 375-382, 1998.

[12] CHOI, B.G., KIM, D.H., JO, C.Y. "Temperature dependence of MC decomposition behavior in Ni-base superalloy GTD-111", Materials Science and Engineering A., v. 478, pp. 329-335, 2008.

[13] CORTEZ, P.M.A. "Estudo microestrutural da superliga de níquel GTD-111 durante o seu envelhecimento e após tratamentos térmicos de rejuvenescimento", Dissertação de M.Sc., Fac. Eng. - Universidade do Porto, Portugal. 2014.

[14] WANGYAO, P., KRONGTONG, V., TUENGSOOK, P., et al., "The relationship between reheattreatment and hardness behavior of cast nickel superalloy, GTD-111", Journal of Materials and Minerals, v.16, n.1 pp.55-62, 2006.

[15] CHERUVU, N. S., SWAMINATHAN, V.P. "Recovery of microstructure and mechanical properties of service run GTD-111 DS buckets", International Gas Turbine \& Aeroengine Congress \& Exhibition. Indianapolis, AMSE, 1999.

[16] TURAZI, A. "Metodologia de avaliação de grau de degradação e rejuvenescimento de superligas à base de níquel", Tese de D.Sc., UFSC, Florianópolis, SC, Brasil, 2014.

[17] PADILHA, A.F. Materiais de engenharia, microestrutura e propriedades. Hemus. Curitiba - PR, 2000.

\section{ORCID}

André Lucas Merini

https://orcid.org/0000-0002-1553-4115

Carlos Augusto Silva Oliveira

http://orcid.org/0000-0002-7096-9066 